\title{
Enhancing $\mathrm{Si}_{3} \mathrm{~N}_{4}$ Waveguide Nonlinearity with Heterogeneous Integration of Few-Layer $\mathrm{WS}_{2}$
}

Yuchen Wang, ${ }^{*}{ }^{\perp}$ Vincent Pelgrin, ${ }^{\perp}$ Samuel Gyger, Gius Md Uddin, Xueyin Bai, Christian Lafforgue, Laurent Vivien, Klaus D. Jöns, Eric Cassan, and Zhipei Sun*

Cite This: ACS Photonics 2021, 8, 2713-2721

Read Online
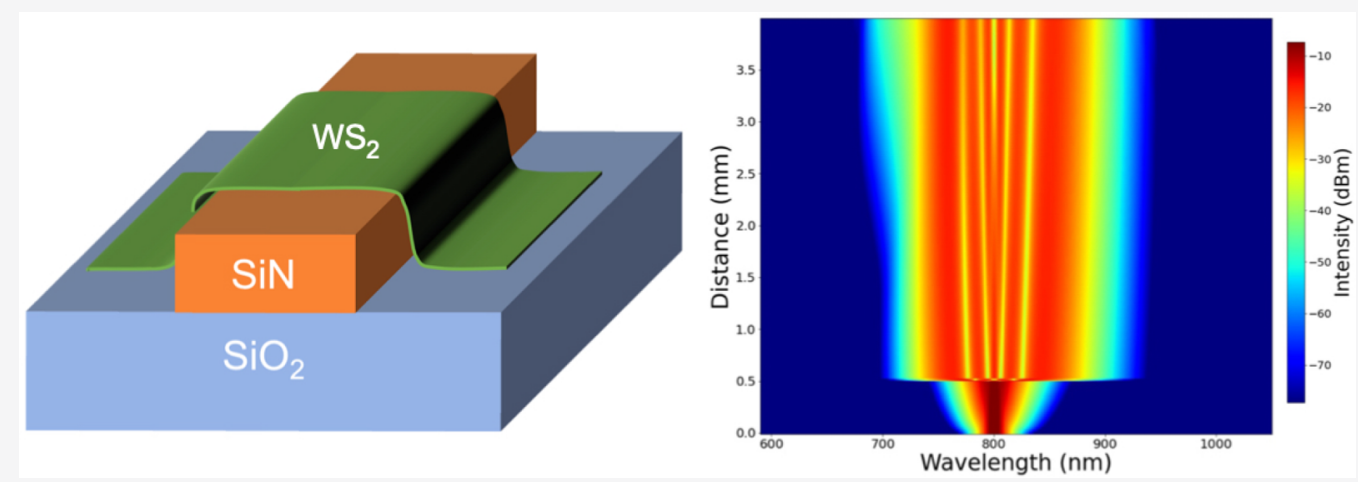

ABSTRACT: The heterogeneous integration of low-dimensional materials with photonic waveguides has spurred wide research interest. Here, we report on the experimental investigation and the numerical modeling of enhanced nonlinear pulse broadening in silicon nitride waveguides with the heterogeneous integration of few-layer $\mathrm{WS}_{2}$. After transferring a few-layer $\mathrm{WS}_{2}$ flake of $\sim 14.8 \mu \mathrm{m}$ length, the pulse spectral broadening in a dispersion-engineered silicon nitride waveguide has been enhanced by $\sim 48.8 \%$ in bandwidth. Through numerical modeling, an effective nonlinear coefficient higher than $600 \mathrm{~m}^{-1} \mathrm{~W}^{-1}$ has been retrieved for the heterogeneous waveguide indicating an enhancement factor of larger than 300 with respect to the pristine waveguide at a wavelength of $800 \mathrm{~nm}$. With further advances in two-dimensional material fabrication and integration techniques, on-chip heterostructures will offer another degree of freedom for waveguide engineering, enabling high-performance nonlinear optical devices, such as frequency combs and quantum light sources.

KEYWORDS: low-dimensional materials, silicon photonics, integrated nonlinear optics, hybrid photonic waveguides, ultrafast optics

$\mathrm{F}$ ollowing recent innovations in integrated optical frequency comb sources and their spectroscopy techniques, breakthroughs have been demonstrated in many fields of study, including precision spectroscopy, ${ }^{1,2}$ low-noise frequency synthesis, ${ }^{3}$ distance ranging, ${ }^{4}$ and quantum light sources. ${ }^{5}$ At the moment, two techniques are among the most frequently used for comb generation: the supercontinuum generation (SCG) process in nonlinear fibers or waveguides pumped with femtosecond mode-locked lasers and the four-wave mixing (FWM) process in microresonators pumped with continuouswave lasers. The integration and the miniaturization of such versatile and powerful broadband coherent light sources with these two techniques have attracted tremendous endeavors, leading to great successes in the development of soliton microcombs, ${ }^{6,7}$ on-chip mode-locked lasers, ${ }^{8}$ and octavespanning supercontinuum generation (SCG). ${ }^{9}$ It is worth mentioning that monolithic and low-cost chip-scale optical frequency combs are already on the verge of reality and envisioned to have a strong impact on both fundamental science and industrial applications.
To take advantage of mature infrastructures and technologies in the CMOS integrated circuit fabrication industry, silicon and silicon nitride $\left(\mathrm{Si}_{3} \mathrm{~N}_{4}\right.$, abbreviated as $\left.\mathrm{SiN}\right)$ are considered among the most convenient materials for integrated photonic devices. Although silicon possesses excellent properties for on-chip comb generation based on both FWM and selfphase modulation (SPM) thanks to its strong third-order nonlinearity, it is limited in power-scaling by the two-photon absorption (TPA) and the free-carrier absorption (FCA) in the near-infrared (near-IR) region between $\sim 1.1$ and $2 \mu \mathrm{m} .{ }^{10}$ The limitation due to FCA can be mitigated by the reverse-biasing of a waveguide integrated with a p-i-n junction. ${ }^{11}$ However, the

Received: May 24, 2021

Published: September 3, 2021 


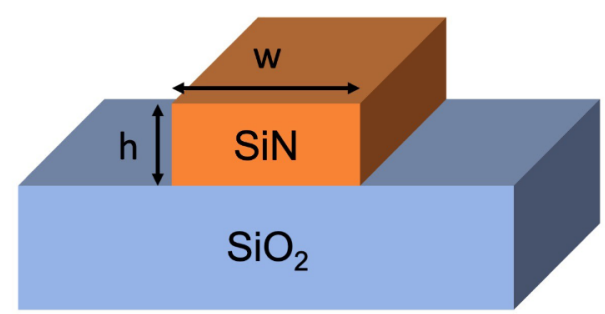

(a)

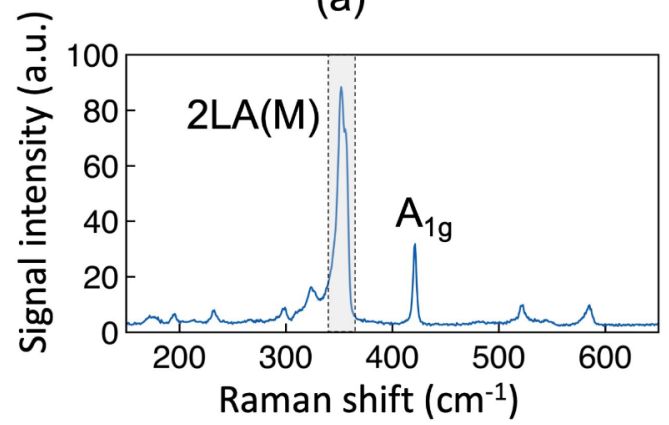

(c)

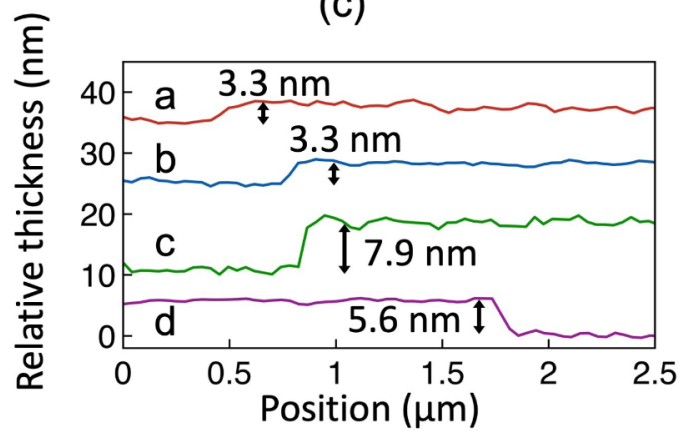

(e)

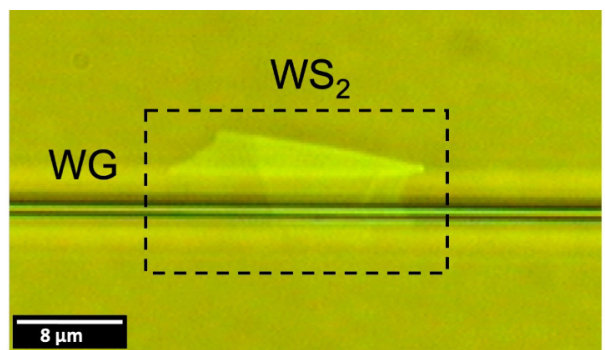

(b)

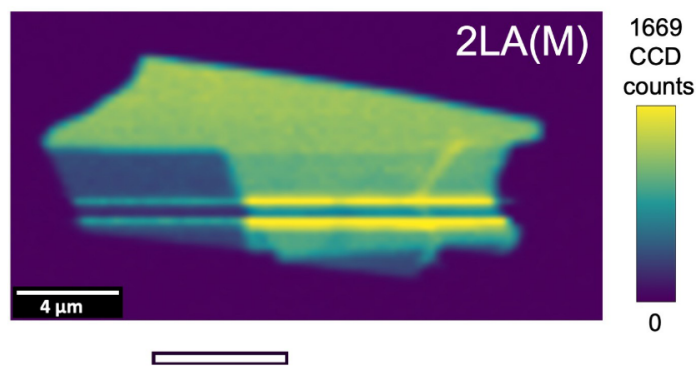

(d)

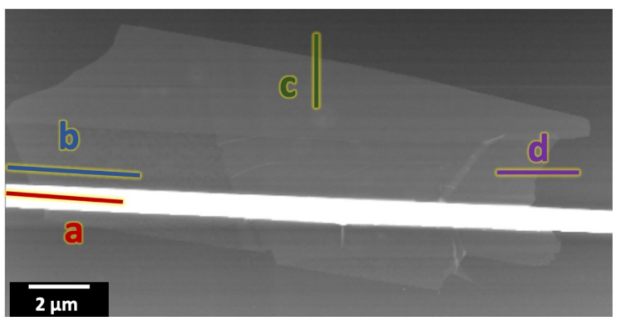

(f)

Figure 1. Heterogeneous waveguide structure: (a) the waveguide cross-section geometry, (b) the transferred WS 2 flake on the waveguide (WG), (c) the Raman spectrum of the $\mathrm{WS}_{2}$ flake, (d) the Raman signal intensity map of the heterogeneous structure, integrated around the characteristic 2LA peak of $\mathrm{WS}_{2}$, (e) the relative thicknesses of the linear AFM scans along the marked paths, and (f) the AFM map of the heterogeneous waveguide with markings of paths for linear scans.

limitation arising from TPA cannot be easily overcome. In comparison, $\mathrm{SiN}$ does not suffer from TPA in the same spectral region thanks to its larger bandgap, but it exhibits a weaker Kerr coefficient $\left(n_{2}=\sim 2.6 \times 10^{-19} \mathrm{~m}^{-2} \mathrm{~W}^{-1}\right.$ as compared to $\sim 4.5 \times 10^{-18} \mathrm{~m}^{-2} \mathrm{~W}^{-1}$ for $\mathrm{Si}$, at $\left.1550 \mathrm{~nm}\right) .{ }^{12}$ Therefore, a trade-off needs to be taken into account while engineering these devices for optimized performance.

With rapidly increasing interest in two-dimensional (2D) layered materials, many of their peculiar properties have been observed and investigated. In particular, some $2 \mathrm{D}$ materials exhibit large nonlinearities, several orders of magnitude larger than conventional bulk nonlinear materials. ${ }^{13-15}$ Considering their minuscule dimensions down to the atomic level, they have great potential for integration with the $\mathrm{SiN}$ photonic platform for increasing its nonlinearities. Some of the most intensively studied 2D materials, such as graphene, ${ }^{16-21}$ graphene oxide, ${ }^{22,23}$ and $\mathrm{MoS}_{2}{ }^{24-26}$ have been investigated for integration with silicon photonic waveguides to improve third-order nonlinear interactions, including SPM and FWM. Enhancements of FWM efficiency up to $7.3 \mathrm{~dB}$ in a $\mathrm{SiN}$ waveguide integrated with graphene oxide $\mathrm{e}^{22}$ and $4 \mathrm{~dB}$ in a silicon waveguide integrated with $\mathrm{MoS}_{2}{ }^{27}$ have been successfully demonstrated. Recently, the heterogeneous integration of $\mathrm{GaS}^{28}$ thin-flakes with $\mathrm{SiN}$ microring resonators has been demonstrated, showing a 5-fold improvement of Kerr coefficient. In comparison to the aforementioned 2D materials, $\mathrm{WS}_{2}$ has an intriguing combination of optical properties, including good transparency and a strong nonlinearity in the near-infrared wavelength range, making it a potential choice for on-chip nonlinear optics, especially in the conventional telecom windows at $\sim 1.5 \mu \mathrm{m}$. $^{15,24,29}$

In this Article, we report on the enhanced on-chip pulse broadening at $800 \mathrm{~nm}$ through SPM in a near-zero normal dispersion regime by the integration of few-layer $\mathrm{WS}_{2}$ on $\mathrm{SiN}$ waveguides. The resulting pulse full-width at half-maximum (fwhm) bandwidth has been improved by $\sim 48.8 \%$ using a fewlayer $\mathrm{WS}_{2}$ flake of $\sim 14.8 \mu \mathrm{m}$ in length. Based on the experimental results, a numerical model has been developed for the $\mathrm{WS}_{2}-\mathrm{SiN}$ heterogeneous waveguide structure, from which an enhancement in the waveguide effective Kerr coefficient of a factor of larger than 300 is retrieved at the wavelength of $800 \mathrm{~nm}$. Thanks to the parallel orientation of the flake with the waveguide transverse electric (TE) mode, the inplane Kerr coefficient of the few-layer $\mathrm{WS}_{2}$ is estimated from our mode overlap model to be $\sim 2.18 \times 10^{-15} \mathrm{~m}^{2} \mathrm{~W}^{-1}$, which is comparable to previously reported values. ${ }^{29}$ These exper- 
imental results prove the intriguing potential of $2 \mathrm{D}$ material heterogeneous waveguides for low-loss and high-efficiency onchip nonlinear optical circuits for frequency comb synthesis and entangled photon pair generation for quantum information processing.

\section{RESULTS AND DISCUSSION}

In this experiment, a few-layer $\mathrm{WS}_{2}$ flake is integrated on a ridged waveguide structure of $760 \mathrm{~nm}$ width (Figure 1a), as shown in the optical microscope image in Figure 1b. The heterogeneous structure is characterized with a scanning micro-Raman spectrometer. Using an excitation laser at 532 $\mathrm{nm}$, the Raman mapping of the heterogeneous structure is performed with a spatial resolution of $500 \mathrm{~nm}$. By summing the Raman spectra signals from $\sim 348$ to $358 \mathrm{~cm}^{-1}$ and from $\sim 417$ to $424 \mathrm{~cm}^{-1}$ (Figure 1c), the spectral intensities of the $2 \mathrm{LA}(\mathrm{M})$ peak and the $\mathrm{A}_{1 \mathrm{~g}}$ peak of $\mathrm{WS}_{2}$ can be obtained, respectively. The integrated $2 \mathrm{LA}$ peak signal mapping is shown in Figure 1d. Consistently, it is clearly visible that the thicker the flake the more intense is the Raman signal. The thickness of the flake is also measured using an atomic force microscope (AFM), as shown in Figure 1e,f, where the waveguide has a height of $330 \mathrm{~nm}$; therefore, it appears saturated in the grayscale image (Figure 1f). The flake covering the waveguide has a region of $\sim 3.3 \mathrm{~nm}$ thickness $(\sim 6.5 \mu \mathrm{m}$ overlap $)$, a region of $\sim 7.9 \mathrm{~nm}$ thickness $(\sim 6.3 \mu \mathrm{m}$ overlap $)$, and a region of $\sim 5.6$ $\mathrm{nm}$ thickness $(\sim 2 \mu \mathrm{m}$ overlap). As a result, the average thickness is $\sim 5.57 \mathrm{~nm}$, and the total overlap length is $\sim 14.8$ $\mu \mathrm{m}$.

The experimental setup of waveguide characterization is shown in Figure 2. A mode-locked Ti:sapphire laser operating

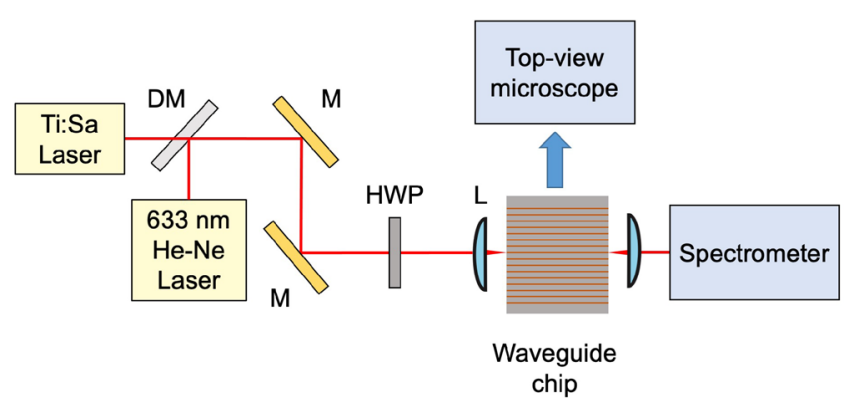

Figure 2. Schematics of the experimental setup for waveguide characterization: DM, dichroic mirror; $\mathrm{M}$, gold mirrors; HWP, halfwave plate; L, aspheric lenses; Ti:Sa laser, Ti:sapphire laser.

at $\sim 84.5 \mathrm{MHz}$ repetition rate with a pulse duration of $\sim 100 \mathrm{fs}$ and a maximum average power of $300 \mathrm{~mW}$ is used as the pump source for nonlinear optical measurements. The pump power is controlled using a variable neutral density filter. The beam from the pump laser passes through a dichroic mirror (longpass filter with a cutoff wavelength of $750 \mathrm{~nm}$ ) and is then steered by a couple of gold mirrors before being focused by an aspheric lens of $1.45 \mathrm{~mm}$ focal length into the waveguides. A continuous-wave (CW) $\mathrm{He}-\mathrm{Ne}$ laser emitting at $633 \mathrm{~nm}$ is aligned to the pump laser beam using the dichroic mirror to aid the waveguide coupling procedure and to perform passive waveguide loss measurements.

Using four spiral waveguides with different lengths on the same chip, the waveguide propagation loss is estimated to be around $1.12 \mathrm{~dB} / \mathrm{mm}$, while the coupling loss is retrieved to be around $5.86 \mathrm{~dB}$ (more details in the Supporting Information).
With the addition of the $\mathrm{WS}_{2}$ flake, additional losses of $\sim 0.91$ and $0.02 \mathrm{~dB}$ have been measured at 633 and $800 \mathrm{~nm}$, respectively. The higher loss measured at $633 \mathrm{~nm}$ is attributed to the higher absorption coefficient of $\mathrm{WS}_{2}$ at that wavelength due to the linear absorption. ${ }^{30}$ Considering the overlap length of the flake, this leads to an additional propagation loss of $\sim 1.35 \mathrm{~dB} / \mathrm{mm}$ at $800 \mathrm{~nm}$. This result compares favorably to former reports of the additional propagation losses due to the integration of $2 \mathrm{D}$ materials in waveguide structures, for example, $2.05 \mathrm{~dB} / \mathrm{mm}$ in a graphene oxide/silicon heterogeneous waveguide $\mathrm{w}^{23}$ and $52 \mathrm{~dB} / \mathrm{mm}$ in a graphene/silicon heterogeneous waveguide. ${ }^{20}$

The nonlinear pulse propagation in the waveguides is first characterized without the integration of $\mathrm{WS}_{2}$. According to the design of the waveguide structure (details in Methods), the waveguide in this experiment has a minimum of integrated dispersion near to the pump wavelength of $800 \mathrm{~nm}$ (Figure $3 a)$, which makes it an ideal choice for characterizing the pulse broadening through the SPM process. The integrated dispersion can be expressed as $D=\sum_{k=2}^{n}\left(\beta_{k} / k !\right)$, where $\beta_{k}$ is the $k^{\text {th }}$ order dispersion. ${ }^{31}$ By controlling the orientation of a half-wave plate, the TE mode of the waveguide is excited (details in the Supporting Information). The pump pulse spectrum is characterized to have a fwhm of $\sim 13 \mathrm{~nm}$. After the nonlinear propagation in the $4 \mathrm{~mm}$ long waveguide, the fwhm of the output pulses increased to $\sim 70.9 \mathrm{~nm}$ at an injected peak pump power of $\sim 1.35 \mathrm{~kW}$ (see Figure $3 \mathrm{~b} ; \sim 123.6 \mathrm{~nm}$ at -20 $\mathrm{dB}$ level). Further increasing the injected peak pump power to $\sim 2.7 \mathrm{~kW}$, the fwhm of the output pulses broadened to $\sim 97.9$ $\mathrm{nm}$ (as shown in Figure 3c; $\sim 163.5 \mathrm{~nm}$ at $-20 \mathrm{~dB}$ level).

The same waveguide is then used for the heterogeneous integration. After the successful deposition of the few-layer $\mathrm{WS}_{2}$ flake (Figure $1 \mathrm{~b}$ ), the heterogeneous $\mathrm{WS}_{2}-\mathrm{SiN}$ waveguide produces a much broader spectrum under the same excitation conditions. The comparison between the spectra before and after the $\mathrm{WS}_{2}$ integration at two different pump power levels is shown in Figure $3 \mathrm{~b}, \mathrm{c}$. At $\sim 1.35 \mathrm{~kW}$ of pump peak power, the fwhm of the output pulses increases to $\sim 105.5$ $\mathrm{nm}(\sim 177.3 \mathrm{~nm}$ at $-20 \mathrm{~dB}$ level $)$. At $\sim 2.7 \mathrm{~kW}$ of peak power, the spectrum is further broadened to a fwhm bandwidth of $\sim 133.5 \mathrm{~nm}$ (from $\sim 730.0$ to $863.5 \mathrm{~nm}$ ) and covering a bandwidth of $\sim 228.1 \mathrm{~nm}$ at $-20 \mathrm{~dB}$ level (from $\sim 672.7$ to $900.8 \mathrm{~nm}$ ). As a result, with the addition of the few-layer $\mathrm{WS}_{2}$ flake, the fwhm of output pulses increased by $\sim 48.8 \%$ at $\sim 1.35$ $\mathrm{kW}$ pump power and $\sim 36.4 \%$ at $\sim 2.7 \mathrm{~kW}$ pump power. These results translate into a maximum pulse broadening factor of $\sim 17.5$ (WS $_{2}$ covered waveguide pumped at $\sim 2.7 \mathrm{~kW}$ pulse peak power), the $13 \mathrm{~nm}$ wide input pulses broadens to $\sim 228$ $\mathrm{nm}$ in the heterogeneous $\mathrm{WS}_{2}-\mathrm{SiN}$ waveguide of $4 \mathrm{~mm}$ total length. This is also a significant improvement compared to previously demonstrated on-chip pulse broadening/compression results. ${ }^{32}$

For a more straightforward comparison of the results, we introduce an enhancement factor $k$ of the waveguide nonlinearity:

$$
k=\frac{\gamma_{\text {hybrid }}}{\gamma_{\mathrm{WG}}}
$$

where $\gamma_{\text {hybrid }}$ is the effective nonlinear coefficient of the heterogeneous structure and $\gamma_{\mathrm{WG}}$ is the effective nonlinear coefficient of the pristine waveguide. 

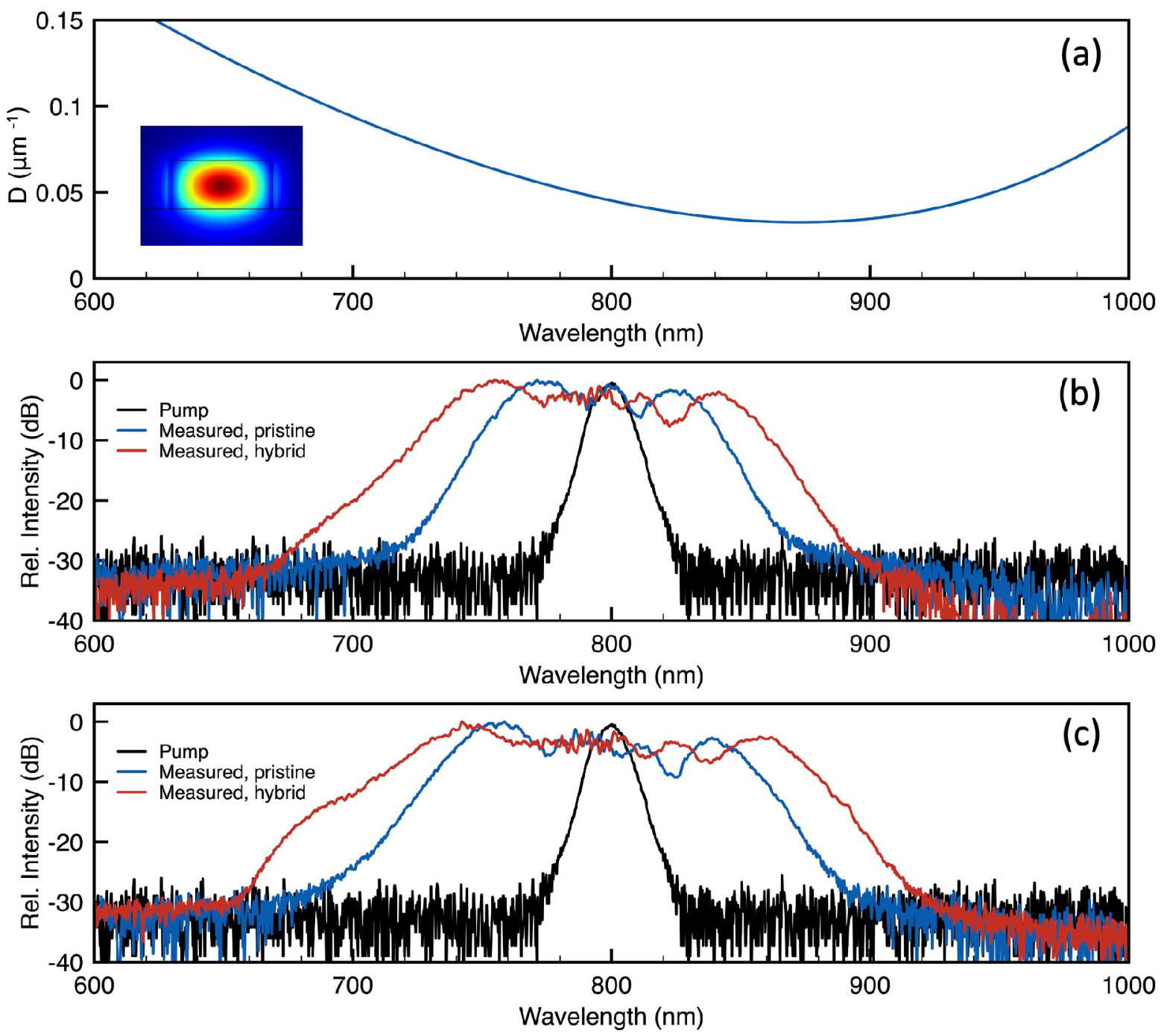

Figure 3. Dispersion properties and pulse broadening effects in the nonlinear waveguides. (a) Integrated dispersion of the TE mode of the pristine $\mathrm{SiN}$ waveguide with a width of $760 \mathrm{~nm}$ (inset: the calculated WG TE-mode profile at $800 \mathrm{~nm}$ ); (b) measured pulse spectra of the pristine WG (blue) and the heterogeneous WG (red) at $1.35 \mathrm{~kW}$ pump peak power (input pump pulse spectrum shown in black); (c) measured pulse spectra of the pristine WG (blue) and heterogeneous WG (red) at $2.7 \mathrm{~kW}$ pump peak power (input pump pulse spectrum shown in black).

After constructing a numerical model for the heterogeneous waveguide, numerical simulations of the nonlinear pulse propagation in the heterogeneous waveguide are carried out for the best fitting of the experimental results (shown in Figure 4; more details in Methods). The model takes into account the measured dimension of the $\mathrm{WS}_{2}$ flake (with a length of $\sim 14.8$ $\mu \mathrm{m}$ and a thickness of $\sim 5.57 \mathrm{~nm}$ ) and its location along the waveguide (at roughly $0.5 \mathrm{~mm}$ from the input facet). By adjusting a set of two parameters, the effective nonlinear coefficient and the TPA coefficient of the heterogeneous waveguide, the output pulse spectra can be recreated with respect to the measured results. In Figure $4 c-f$, the false-color maps illustrate the evolution of the spectrum of the pulses traveling along the nonlinear waveguides. The enhancement effect introduced by the $\mathrm{WS}_{2}$ flake is rather evident considering the small flake length of $14.8 \mu \mathrm{m}$ (which is located at $z=0.5$ $\mathrm{mm}$ from the input). The large nonlinearity in the heterogeneous section largely anticipates the saturation of the nonlinear broadening effect which does not happen in the pristine $\mathrm{SiN}$ waveguide with a length of $4 \mathrm{~mm}$.
At a pump power of $1.35 \mathrm{~kW}$, an effective nonlinear coefficient $\gamma_{\text {hybrid }}$ of $\sim 650 \mathrm{~W}^{-1} \mathrm{~m}^{-1}$ is retrieved for the heterogeneous waveguide. While at $2.7 \mathrm{~kW}$ pump power, the retrieved effective nonlinear coefficient is slightly lower at $\sim 600 \mathrm{~W}^{-1} \mathrm{~m}^{-1}$. The lower effective nonlinear coefficient retrieved at higher pump power is most likely due to the limitation of the broadening effect arising from the stronger linear absorption in $\mathrm{WS}_{2}$ at wavelengths close to $600 \mathrm{~nm}$. For both power levels, the TPA coefficient of $\mathrm{WS}_{2}$ that provides the best fitting is $\sim 158 \mathrm{~cm} / \mathrm{GW}$, which agrees with the experimentally measured values of $\sim 525 \pm 205 \mathrm{~cm} / \mathrm{GW}$ reported in ref 33. As a result, the heterogeneous waveguide shows a significant improvement compared to the pristine SiN waveguide $\left(\gamma_{\mathrm{SiN}}=\sim 1.75 \mathrm{~W}^{-1} \mathrm{~m}^{-1}\right)$, with enhancement factors $k$ of $\sim 371.4$ and $\sim 342.9$ for input pump powers of 1.35 and 2.7 $\mathrm{kW}$, respectively. With the retrieved real and imaginary parts of the effective third-order nonlinear coefficient at $1.35 \mathrm{~kW}$ pump power, the nonlinear figure-of-merit of the heterogeneous waveguide is estimated to be $\sim 1.7$ using the definition in ref 34. 

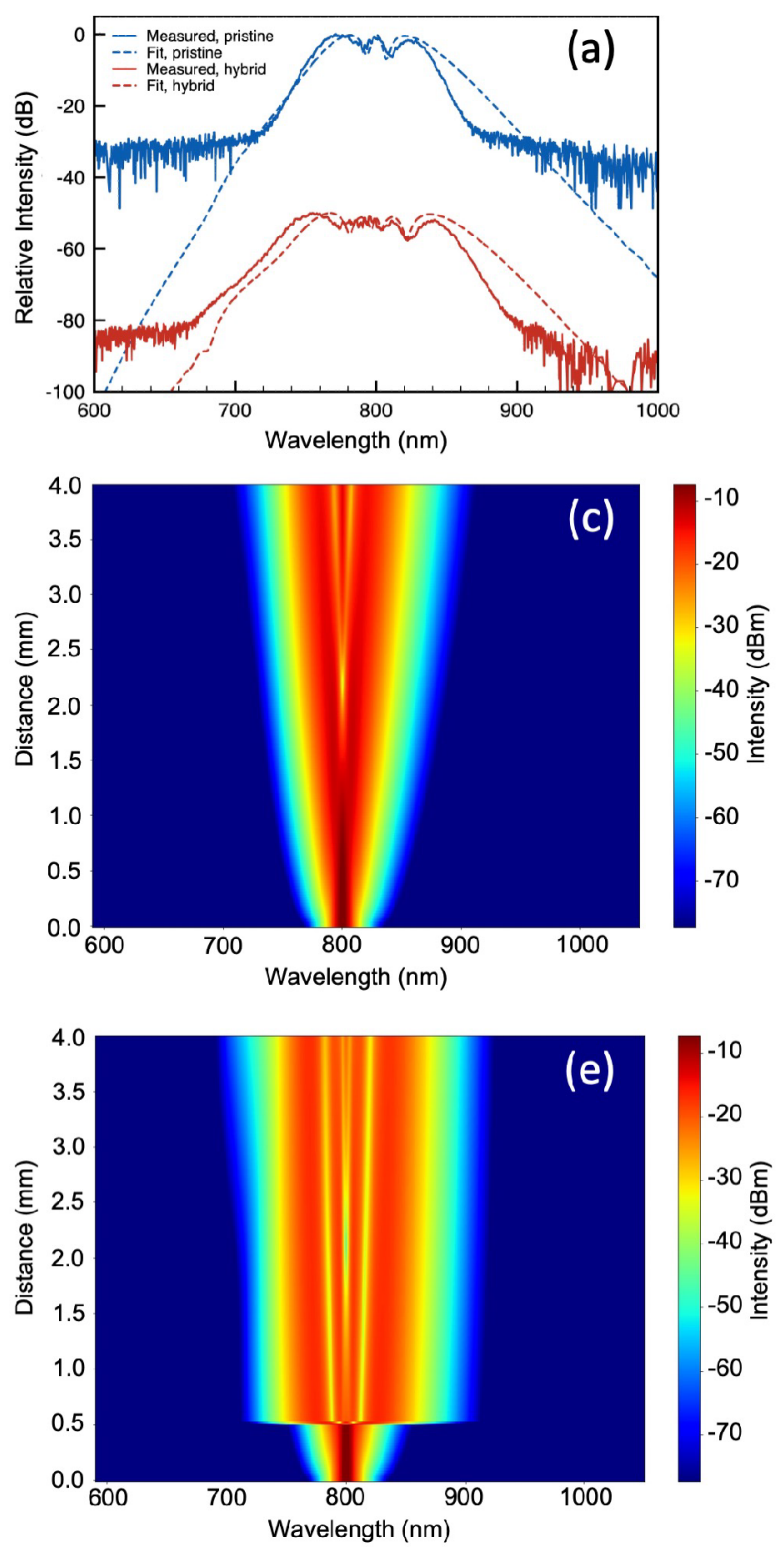
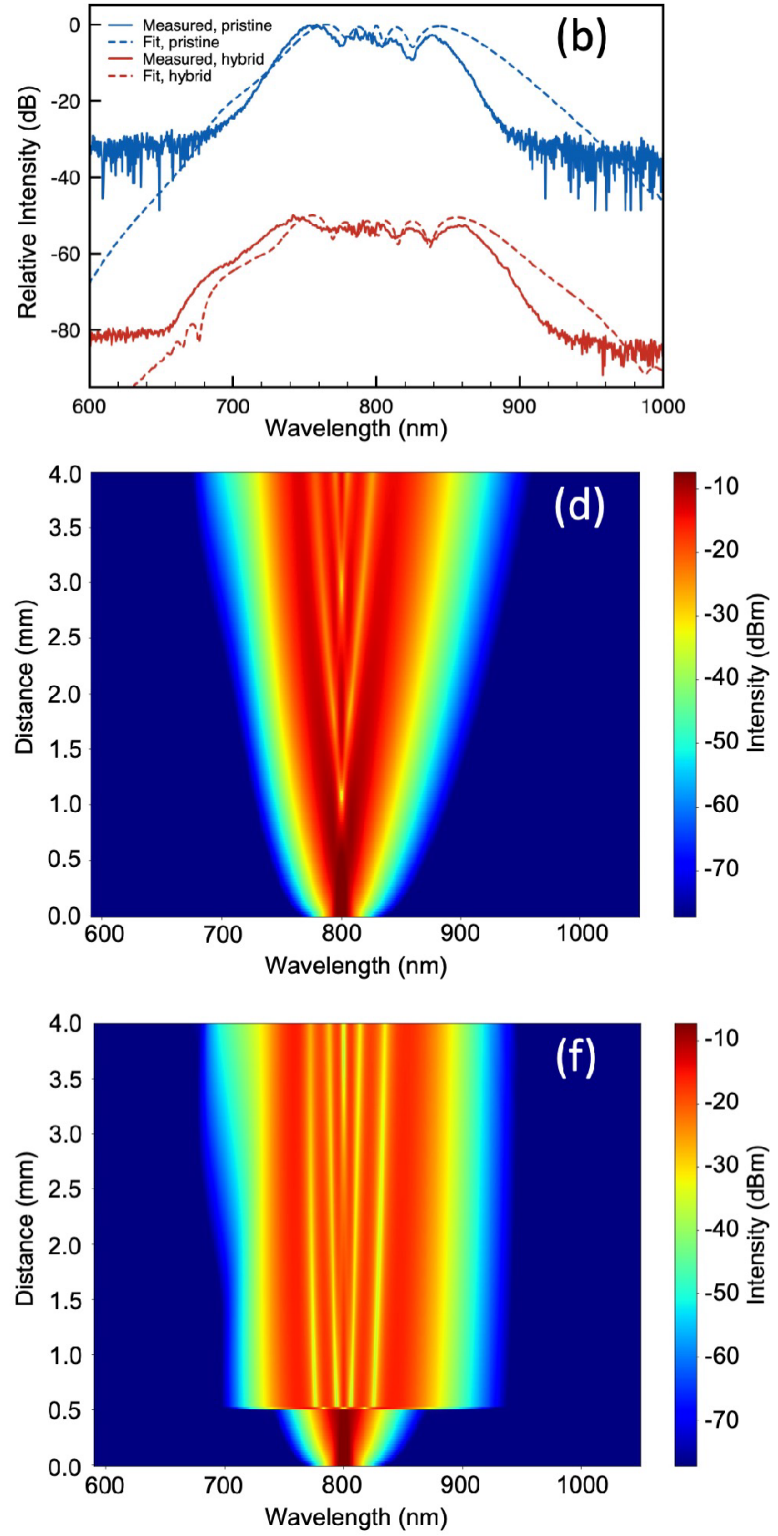

Figure 4. Simulated fitting curves for the pulse broadening in the waveguides: (a) measured pulse spectra (solid line) and simulated fitting spectra (dashed line) at $1.35 \mathrm{~kW}$ input peak pump power of the pristine WG (blue) and the heterogeneous WG (red); (b) measured pulse spectra (solid line) and simulated fitting spectra (dashed line) at $2.7 \mathrm{~kW}$ input peak pump power of the pristine WG (blue) and heterogeneous WG (red); and simulated pulse propagation false-color map of (c) the pristine waveguide at $1.35 \mathrm{~kW}$ input peak power, (d) the pristine waveguide at $2.7 \mathrm{~kW}$ input peak power, (e) the heterogeneous waveguide at $1.35 \mathrm{~kW}$ input peak power, and (f) the heterogeneous waveguide at $2.7 \mathrm{~kW}$ input peak power.

The results from this experiment are summarized and compared with previously reported experimental results of $2 \mathrm{D}$ material heterogeneous WG structures in Table 1. Currently, the largest nonlinear coefficients in channel waveguides are demonstrated with graphene-Si heterogeneous structures, with effective nonlinear coefficients exceeding $1000 \mathrm{~m}^{2} \mathrm{~W}^{-1}{ }^{18,21}$ However, graphene also introduces high propagation losses. On the contrary, as a dielectric, graphene oxide (GO) does not absorb linearly and shows a large Kerr coefficient a few orders of magnitude higher than $\mathrm{Si}^{35}$ Although transition metal dichalcogenides, such as $\mathrm{MoS}_{2}$ and $\mathrm{WS}_{2}$, do not offer Kerr coefficients as high as GO, they provide better versatilities, such as the access to second-order nonlinearity with their monolayers and complex optoelectronic devices for their semiconducting nature. Thanks to the optimization of the waveguide dispersion profile and material properties, the pulse broadening effects obtained in the $\mathrm{WS}_{2}-\mathrm{SiN}$ heterogeneous waveguide in this work is much more significant than the $\mathrm{MoS}_{2}-\mathrm{Si}$ heterogeneous waveguide previously reported (spectral bandwidth of $\sim 2.2 \mathrm{~nm}$ at $-20 \mathrm{~dB}$ level and a difference of $\sim 10 \%$ between the heterogeneous waveguide and the pristine waveguide). ${ }^{36}$ The effective nonlinear coefficients of the $\mathrm{WS}_{2}-\mathrm{SiN}$ structure in this work $\left(\gamma_{\text {eff }}=\sim 600 \mathrm{~m}^{-1} \mathrm{~W}^{-1}\right)$ also reaches the level achieved with GO-Si/SiN structures $\left(\gamma_{\text {eff }}\right.$ $\left.=167 \mathrm{~m}^{-1} \mathrm{~W}^{-1}\right) .^{22}$

Using the waveguide mode overlap model and the waveguide effective susceptibility model described by eq 4 in the Methods, the Kerr coefficient of the $\mathrm{WS}_{2}$ flake can be retrieved. Under the condition of $1.35 \mathrm{~kW}$ pump power, the Kerr coefficient $n_{2}$ of the few-layer $\mathrm{WS}_{2}$ flake is estimated to be $\sim 2.18 \times 10^{-15} \mathrm{~m}^{2} \mathrm{~W}^{-1}$ at $800 \mathrm{~nm}$. Instead, at $2.7 \mathrm{~kW}$ pump power, the estimated Kerr coefficient is lower, at $\sim 2.01 \times$ 
Table 1. Summary of the Experimental Results of Nonlinear Pulse Broadening and the Numerically Retrieved Nonlinear Optical Properties of the Waveguide Structures ${ }^{a}$

\begin{tabular}{|c|c|c|c|c|c|c|c|c|}
\hline structure & $\lambda(\mathrm{nm})$ & $P_{\mathrm{p}}(\mathrm{kW})$ & fwhm (nm) & $\gamma_{\mathrm{eff}}\left(\mathrm{m}^{-1} \mathrm{~W}^{-1}\right)$ & $n_{2}\left(\mathrm{~m}^{2} \mathrm{~W}^{-1}\right)$ & $K$ & $\alpha_{2}(\mathrm{~cm} / \mathrm{GW})$ & ref \\
\hline \multirow{2}{*}{ pristine $\mathrm{SiN}$} & & 1.35 & 70.9 & \multirow{2}{*}{1.75} & \multirow{2}{*}{$3.3 \times 10^{-20}$} & - & - & \multirow{4}{*}{ this work } \\
\hline & & 2.7 & 97.9 & & & - & - & \\
\hline \multirow[b]{2}{*}{$\mathrm{WS}_{2}-\mathrm{SiN}$} & 800 & 1.35 & 105.5 & 650 & $9.5 \times 10^{-19}$ & 371.4 & \multirow[b]{2}{*}{158} & \\
\hline & & 2.7 & 133.5 & 600 & $8.8 \times 10^{-19}$ & 342.8 & & \\
\hline graphene-SiN & & - & - & $2000-6400$ & - & $\sim 10^{3}$ & - & 21 \\
\hline \multirow[t]{2}{*}{ graphene-Si } & & - & - & - & $2 \times 10^{-17}$ & 3 & - & 17 \\
\hline & & - & - & 1542 & - & 9.8 & - & 18 \\
\hline GO-Si & 1550 & - & - & - & $9.6 \times 10^{-17}$ & 16 & - & 23 \\
\hline GO-SiN & & - & - & 167 & $1.5 \times 10^{-17}$ & 5.6 & - & 22 \\
\hline $\mathrm{MoS}_{2}-\mathrm{Si}$ & & - & - & - & $4.7 \times 10^{-15}$ & 5.6 & - & 26 \\
\hline GaS-SiN & & - & - & - & - & 5 & - & 28 \\
\hline
\end{tabular}

${ }^{a} \lambda$, center wavelength of optical characterizations; $P_{\mathrm{p}}$, pump peak power; $\gamma_{\mathrm{eff}}$, effective nonlinear coefficient; $k$, enhancement factor of the effective nonlinear coefficient; $\alpha_{2}$, retrieved TPA coefficient; Si, silicon.

$10^{-15} \mathrm{~m}^{2} \mathrm{~W}^{-1}$. These retrieved values are on the same level as the experimental results of $\sim 1.15 \times 10^{-15} \mathrm{~m}^{2} \mathrm{~W}^{-1}$ measured at $532 \mathrm{~nm}$ using a vectorial two-wave mixing method. ${ }^{29}$

Assisted by the detailed numerical model for the heterogeneous waveguide and the potential for a fully fiberbased alignment-free experimental setup, the heterogeneous integration method could be extended to the characterization of the linear and nonlinear optical responses of lowdimensional materials and their on-chip nonlinear optical circuit applications. Without the requirement of any moving part and free-space alignment, it is more stable and efficient than the conventional z-scan method. While exciting the waveguide transverse magnetic mode, the out-of-plane components of optical properties can also be easily accessed, which is prohibited in the $\mathrm{z}$-scan method at normal incidence.

In conclusion, we have demonstrated the enhancement of nonlinear pulse broadening in a SiN waveguide integrated with few-layer $\mathrm{WS}_{2}$. In a condition of normal dispersion with a nearzero GVD, the enhancement of waveguide Kerr nonlinearity has been characterized with a mode-overlap model. From the measured results, an enhancement in the effective third-order nonlinear coefficient of a factor of larger than 300 has been retrieved, as compared to the pristine $\mathrm{SiN}$ waveguide. The significant pulse broadening demonstrated in the heterogeneous waveguides could find immediate applications in on-chip SCG and pulse compression. With a mode overlap and waveguide effective susceptibility model, the Kerr coefficient of few-layer $\mathrm{WS}_{2}$ at $800 \mathrm{~nm}$ was retrieved to be $\sim 2.18 \times 10^{-15} \mathrm{~m}^{2}$ $\mathrm{W}^{-1}$. With the rapid advance in the direct growth of transition metal dichalcogenides employing conventional chemical vapor deposition (CVD) techniques, ${ }^{37}$ the fabrication of heterogeneous waveguide structures can also be compatible with the existing CMOS manusfacturing technologies and infrastructures. With the precise control of the number of layers and the flake dimension in the direct CVD growth, the waveguide effective nonlinearity can therefore be engineered. The heterogeneous integration of $2 \mathrm{D}$ materials is shown to be a viable strategy for enhancing the nonlinearity of conventional SiN waveguide platform, therefore, increasing the efficiency of nonlinear frequency conversion processes such as SCG, FWM, and parametric down-conversion.

\section{METHODS}

Fabrication of the Heterogeneous Waveguide. The waveguide chip used in this work is fabricated from a commercial low-pressure chemical-vapor-deposited (LPCVD) silicon nitride wafer with a nitride thickness of $\sim 330 \mathrm{~nm}$ and an underlying silicon oxide layer with a thickness of $\sim 3.3 \mu \mathrm{m}$ (Rogue Valley Microdevices). The waveguides are patterned using electron beam lithography with negative resist then followed by fluorine-based reactive ion etching. The waveguides have a ridged cross-sectional structure, as illustrated in Figure 1a, with a height of $\sim 330 \mathrm{~nm}$ and a width ranging from 540 to $840 \mathrm{~nm}$. The width of $760 \mathrm{~nm}$ is then chosen after experimental confirmation, for an optimized dispersion profile. A s-shape displacement is made in the middle of the waveguide by two $90^{\circ}$ turns, with a radius of curvature of $50 \mu \mathrm{m}$, to reduce stray light collection at the output end.

For the heterogeneous integration of $\mathrm{WS}_{2}$ few-layers with high optical quality, the mechanical exfoliation method is used for sample preparation. Mediated by polydimethylsiloxane stamps, exfoliated thin flakes are deterministically transferred onto the upper waveguide surface using a commercial nanomaterial transfer system (HQ Graphene). The entire exfoliation and transfer processes are performed in a cleanroom environment to avoid any contamination from undesired particles. By controlling the exfoliation process, a few-layer $\mathrm{WS}_{2}$ flake is isolated and then transferred on top of the waveguide without contaminating nearby waveguides.

Measurement Apparatus. For the pulse broadening measurements, a Ti:sapphire femtosecond laser (SpectraPhysics MaiTai) emitting pulses at $800 \mathrm{~nm}$ central wavelength operating at a repetition rate of $\sim 84.5 \mathrm{MHz}$ with a pulse duration of $\sim 100 \mathrm{fs}(\sim 13 \mathrm{~nm}$ fwhm bandwidth) at a maximum average power of $\sim 300 \mathrm{~mW}$ is used as the pump source. The laser powers are measured with a photodiode-based power meter (Ophir) while the spectra of the laser pulses before and after nonlinear propagation are measured with a highsensitivity spectrometer covering the visible to near-IR range (Ocean Optics HR 4000).

Numerical Modeling. For a better understanding of the physical processes behind the nonlinear pulse propagation and the enhancement of the waveguide effective nonlinearity by introducing the $\mathrm{WS}_{2}$ flake, we have conducted detailed mode overlap analyses and nonlinear pulse propagation simulations. 
The heterogeneous waveguide structure was analyzed using a commercial finite difference eigenmodes mode solver (Lumerical). The effect of the introduced $\mathrm{WS}_{2}$ flake can be modeled using its surface conductivity $\sigma_{\mathrm{s}}$ and relative permittivity $\epsilon_{\mathrm{r}}{ }^{38}$ The relative permittivity can be expressed as the sum of $N$ Lorentzian functions:

$$
\epsilon_{\mathrm{r}}=\frac{\epsilon(\omega)}{\epsilon_{0}}=1+\sum_{k=1}^{N} \frac{f_{k}}{\omega_{k}^{2}-\omega^{2}-i \omega \gamma_{k}}
$$

where $\gamma_{k}$ is the oscillator strength, $\omega_{k}$ is the resonance frequency, and $f_{k}$ the spectral width, of the $k^{\text {th }}$ oscillator. These coefficients used in this expression can be extracted from experimental measurements of $\mathrm{WS}_{2} .{ }^{39}$ Then the surface conductivity can be retrieved using the following expression of the dielectric function:

$$
\epsilon(\omega)=\epsilon_{0}\left(1+\frac{i \sigma_{\mathrm{b}}}{\epsilon_{0} \omega}\right)=\epsilon_{0}\left(1+\frac{i \sigma_{\mathrm{s}}}{\epsilon_{0} \omega h_{\text {eff }}}\right)
$$

where $\sigma_{\mathrm{b}}$ is the bulk conductivity of the material and $h_{\mathrm{eff}}$ is the effective thickness of the $\mathrm{WS}_{2}$ flake under consideration $(\sim 5.57$ $\mathrm{nm})$.

After constituting the waveguide cross-section model using the calculated surface conductivity of $\mathrm{WS}_{2}$ and the Sellmeier equations for LPCVD $\mathrm{Si}_{3} \mathrm{~N}_{4}$ and $\mathrm{SiO}_{2},{ }^{25,40}$ the waveguide effective indices are calculated for the TE mode over a wide wavelength range from 600 to $1000 \mathrm{~nm}$. It has been shown that compared to a waveguide with the same geometry without the 2D material, the material introduced little change to the waveguide dispersion. ${ }^{41}$ Therefore, here we assume that the heterogeneous waveguide has the same dispersion operator of the $\mathrm{SiN}$ waveguide before the integration of $\mathrm{WS}_{2}$ flake. The calculated total dispersion operator of a waveguide with a width of $760 \mathrm{~nm}$ is shown in Figure 3a. On the other hand, considering the thickness of the $\mathrm{WS}_{2}$ of $\sim 5.57 \mathrm{~nm}$, the mode field distribution of the heterogeneous waveguide with the $\mathrm{WS}_{2}$ flake can be calculated. Here we consider the average thickness because the third-order nonlinearity of $\mathrm{WS}_{2}$ is shown to depend relatively linearly with the thickness (number of layers). ${ }^{29}$ As a result, an overlap factor of the guided mode with the $\mathrm{WS}_{2}$ flake is calculated to be $\eta=0.45 \%$ using the model reported in ref 41 . In the end, an effective nonlinear susceptibility of the heterogeneous waveguide can be written as the weighted integral of the corresponding tensor susceptibility over the waveguide mode: ${ }^{42}$

$$
\Gamma=\frac{A_{0} \int_{A_{0}} \mathbf{e}^{*}\left(\mathbf{r}_{\perp} ; \omega\right) \chi^{(3)}\left(\mathbf{r}_{\perp}\right) \mathbf{e}\left(\mathbf{r}_{\perp} ; \omega\right) \mathbf{e}^{*}\left(\mathbf{r}_{\perp} ; \omega\right) \mathbf{e}\left(\mathbf{r}_{\perp} ; \omega\right) \mathrm{d} A}{\left(\int_{A_{\infty}} n^{2}\left(\mathbf{r}_{\perp}\right)\left|\mathbf{e}\left(\mathbf{r}_{\perp} ; \omega\right)\right|^{2} \mathrm{~d} A\right)^{2}}
$$

where $A_{0}$ is the area of the waveguide cross-section, $A_{\infty}$ denotes the integration to be performed across the entire transverse plane, $\omega$ is the frequency of the pump light, $\mathbf{e}\left(\mathbf{r}_{\perp} ; \omega\right)$ is the electric field profile of the waveguide mode (for our waveguide, which is uniform along its length, the mode depends only on the transverse coordinate $\left.\mathbf{r}_{\perp}\right), \chi^{(3)}\left(\mathbf{r}_{\perp}\right)$ is the third-order nonlinear susceptibility tensor over the transverse coordinate, and $n\left(\mathbf{r}_{\perp}\right)$ is the refractive index over the transverse coordinate.

Using the calculated waveguide dispersion profile, the nonlinear pulse propagation in the waveguides has been modeled with the generalized nonlinear Schrödinger equation in the frequency domain. ${ }^{31,43,44}$ To better assess the influence of the $\mathrm{WS}_{2}$ flake to the $\mathrm{SiN}$ waveguide, the finite length of the flake has been taken into account. We divide the device into three sections: one heterogeneous section with a $\mathrm{WS}_{2}$ flake between two pristine $\mathrm{SiN}$ waveguides. For the simulation of spectral broadening, we use a transform-limited pump pulse with a duration of $\sim 100$ fs and peak powers close to the estimated power coupled to the waveguide TE mode. Since the TPA coefficient is quite large for $\mathrm{WS}_{2}$ in the wavelength range of this experiment, we also take into account the TPA effect in the simulation. ${ }^{33}$ The SiN has an energy bandgap more than twice the pump photon energy, which is confirmed by the negligible TPA coefficient of $2.9 \times 10^{-8} \mathrm{~cm} / \mathrm{GW}$ measured in our experiment (see Supporting Information). Therefore, in the numerical model, we consider only the TPA coefficient of the $\mathrm{WS}_{2}$ flake. From the simulation results, reasonable fits to the experimental data can be obtained for both the simple waveguide and the heterogeneous waveguide, especially the peak wavelengths of the spectral features created by SPM in the normal dispersion regime (Figure $4 a, b$ ). At the longer wavelength side, the simulation departs slightly from the measured spectrum, most likely due to the limited accuracy in the dispersion profile. From the best fitting results, we can retrieve the effective nonlinear coefficient and the TPA coefficient of the heterogeneous waveguide.

\section{ASSOCIATED CONTENT}

\section{Supporting Information}

The Supporting Information is available free of charge at https://pubs.acs.org/doi/10.1021/acsphotonics.1c00767.

Additional details of waveguide cross section scanning electron microscope images, $\mathrm{WS}_{2}$ flake characterization, waveguide loss characterization, and waveguide twophoton absorption characterization (PDF)

\section{AUTHOR INFORMATION}

\section{Corresponding Authors}

Yuchen Wang - Department of Electronics and Nanoengineering, Aalto University, Espoo 02150, Finland; (1) orcid.org/0000-0002-5815-1755;

Email: yuchen.xindi.wang@gmail.com

Zhipei Sun - Department of Electronics and Nanoengineering and QTF Centre of Excellence, Department of Applied Physics, Aalto University, Espoo 02150, Finland; ๑ orcid.org/0000-0002-9771-5293; Email: zhipei.sun@ aalto.fi

\section{Authors}

Vincent Pelgrin - Department of Electronics and Nanoengineering, Aalto University, Espoo 02150, Finland; Université Paris-Saclay, CNRS, Centre de Nanosciences et de Nanotechnologies, Palaiseau 91120, France

Samuel Gyger - Department of Applied Physics, KTH Royal Institute of Technology, Stockholm 114 28, Sweden; (1) orcid.org/0000-0003-2080-9897

Gius Md Uddin - Department of Electronics and Nanoengineering, Aalto University, Espoo 02150, Finland

Xueyin Bai - Department of Electronics and Nanoengineering, Aalto University, Espoo 02150, Finland; 이이이.org/00000002-2254-5701 
Christian Lafforgue - Université Paris-Saclay, CNRS, Centre de Nanosciences et de Nanotechnologies, Palaiseau 91120, France

Laurent Vivien - Université Paris-Saclay, CNRS, Centre de Nanosciences et de Nanotechnologies, Palaiseau 91120,

France; 이이.orcid.org/0000-0002-2980-7225

Klaus D. Jöns - Institute for Photonic Quantum Systems (PhoQS), Center for Optoelectronics and Photonics Paderborn (CeOPP) and Department of Physics, Paderborn University, 33098 Paderborn, Germany

Eric Cassan - Université Paris-Saclay, CNRS, Centre de Nanosciences et de Nanotechnologies, Palaiseau 91120, France

Complete contact information is available at: https://pubs.acs.org/10.1021/acsphotonics.1c00767

\section{Author Contributions}

${ }^{\perp}$ These authors contributed equally to this work.

\section{Notes}

The authors declare no competing financial interest.

\section{ACKNOWLEDGMENTS}

The authors gratefully acknowledge funding from Academy of Finland (314810, 333982, 336144, and 336818), the Academy of Finland Flagship Programme (320167,PREIN), the European Union's Horizon 2020 research and innovation program (820423, S2QUIP; 965124, FEMTOCHIP), the EU H2020MSCA-RISE-872049 (IPN-Bio), the European Research Council (647342, 834742), and the Agence Nationale de la Recherche (ANR OpticAll).

\section{REFERENCES}

(1) Picqué, N.; Hänsch, T. W. Frequency comb spectroscopy. Nat. Photonics 2019, 13, 146-157.

(2) Lamb, E. S.; Carlson, D. R.; Hickstein, D. D.; Stone, J. R.; Diddams, S. A.; Papp, S. B. Optical-frequency measurements with a Kerr microcomb and photonic-chip supercontinuum. Phys. Rev. Appl. 2018, 9, 024030.

(3) Del'Haye, P.; Coillet, A.; Fortier, T.; Beha, K.; Cole, D. C.; Yang, K. Y.; Lee, H.; Vahala, K. J.; Papp, S. B.; Diddams, S. A. Phasecoherent microwave-to-optical link with a self-referenced microcomb. Nat. Photonics 2016, 10, 516-520.

(4) Suh, M.-G.; Vahala, K. J. Soliton microcomb range measurement. Science 2018, 359, 884-887.

(5) Wang, Y.; Jöns, K. D.; Sun, Z. Integrated photon-pair sources with nonlinear optics. Appl. Phys. Rev. 2021, 8, 011314.

(6) Stern, B.; Ji, X.; Okawachi, Y.; Gaeta, A. L.; Lipson, M. Batteryoperated integrated frequency comb generator. Nature 2018, 562, 401-405.

(7) Shen, B.; Chang, L.; Liu, J.; Wang, H.; Yang, Q.-F.; Xiang, C.; Wang, R. N.; He, J.; Liu, T.; Xie, W.; et al. Integrated turnkey soliton microcombs. Nature 2020, 582, 365-369.

(8) Shtyrkova, K.; Callahan, P. T.; Li, N.; Magden, E. S.; Ruocco, A.; Vermeulen, D.; Kärtner, F. X.; Watts, M. R.; Ippen, E. P. Integrated CMOS-compatible Q-switched mode-locked lasers at $1900 \mathrm{~nm}$ with an on-chip artificial saturable absorber. Opt. Express 2019, 27, 35423556.

(9) Singh, N.; Xin, M.; Vermeulen, D.; Shtyrkova, K.; Li, N.; Callahan, P. T.; Magden, E. S.; Ruocco, A.; Fahrenkopf, N.; Baiocco, $\mathrm{C}$.; et al. Octave-spanning coherent supercontinuum generation in silicon on insulator from $1.06 \mu \mathrm{m}$ to beyond $2.4 \mu \mathrm{m}$. Light: Sci. Appl. 2018, 7, 17131-17131.

(10) Tsang, H. K.; Wong, C.; Liang, T.; Day, I.; Roberts, S.; Harpin, A.; Drake, J.; Asghari, M. Optical dispersion, two-photon absorption and self-phase modulation in silicon waveguides at $1.5 \mu \mathrm{m}$ wavelength. Appl. Phys. Lett. 2002, 80, 416-418.

(11) Turner-Foster, A. C.; Foster, M. A.; Levy, J. S.; Poitras, C. B.; Salem, R.; Gaeta, A. L.; Lipson, M. Ultrashort free-carrier lifetime in low-loss silicon nanowaveguides. Opt. Express 2010, 18, 3582-3591.

(12) Rahim, A.; Ryckeboer, E.; Subramanian, A. Z.; Clemmen, S.; Kuyken, B.; Dhakal, A.; Raza, A.; Hermans, A.; Muneeb, M.; Dhoore, S.; et al. Expanding the Silicon Photonics Portfolio With Silicon Nitride Photonic Integrated Circuits. J. Lightwave Technol. 2017, 35, 639-649.

(13) Autere, A.; Jussila, H.; Dai, Y.; Wang, Y.; Lipsanen, H.; Sun, Z. Nonlinear optics with 2D layered materials. Adv. Mater. 2018, 30, 1705963.

(14) Saynatjoki, A.; Karvonen, L.; Rostami, H.; Autere, A.; Mehravar, S.; Lombardo, A.; Norwood, R. A.; Hasan, T.; Peyghambarian, N.; Lipsanen, H.; Kieu, K.; Ferrari, A. C.; Polini, M.; Sun, Z.; et al. Ultra-strong nonlinear optical processes and trigonal warping in $\mathrm{MoS}_{2}$ layers. Nat. Commun. 2017, 8, 1-8.

(15) Autere, A.; Jussila, H.; Marini, A.; Saavedra, J. R. M.; Dai, Y.; Säynätjoki, A.; Karvonen, L.; Yang, H.; Amirsolaimani, B.; Norwood, R. A.; et al. Optical harmonic generation in monolayer group-VI transition metal dichalcogenides. Phys. Rev. B: Condens. Matter Mater. Phys. 2018, 98, 115426.

(16) Feng, Q.; Cong, H.; Zhang, B.; Wei, W.; Liang, Y.; Fang, S.; Wang, T.; Zhang, J. Enhanced optical Kerr nonlinearity of graphene/ Si hybrid waveguide. Appl. Phys. Lett. 2019, 114, 071104.

(17) Ji, M.; Cai, H.; Deng, L.; Huang, Y.; Huang, Q.; Xia, J.; Li, Z.; $\mathrm{Yu}$, J.; Wang, Y. Enhanced parametric frequency conversion in a compact silicon-graphene microring resonator. Opt. Express 2015, 23, 18679-18685.

(18) Yonezu, Y.; Kou, R.; Nishi, H.; Tsuchizawa, T.; Yamada, K.; Aoki, T.; Ishizawa, A.; Matsuda, N. Evaluation of graphene optical nonlinearity with photon-pair generation in graphene-on-silicon waveguides. Opt. Express 2019, 27, 30262-30271.

(19) Vermeulen, N.; Castelló-Lurbe, D.; Khoder, M.; Pasternak, I.; Krajewska, A.; Ciuk, T.; Strupinski, W.; Cheng, J.; Thienpont, H.; Van Erps, J. Graphene's nonlinear-optical physics revealed through exponentially growing self-phase modulation. Nat. Commun. 2018, $9,1-9$.

(20) Ishizawa, A.; Kou, R.; Goto, T.; Tsuchizawa, T.; Matsuda, N.; Hitachi, K.; Nishikawa, T.; Yamada, K.; Sogawa, T.; Gotoh, H. Optical nonlinearity enhancement with graphene-decorated silicon waveguides. Sci. Rep. 2017, 7, 1-9.

(21) Alexander, K.; Savostianova, N. A.; Mikhailov, S. A.; Kuyken, B.; Van Thourhout, D. Electrically tunable optical nonlinearities in graphene-covered $\mathrm{SiN}$ waveguides characterized by four-wave mixing. ACS Photonics 2017, 4, 3039-3044.

(22) Yang, Y.; Wu, J.; Xu, X.; Liang, Y.; Chu, S. T.; Little, B. E.; Morandotti, R.; Jia, B.; Moss, D. J. Enhanced four-wave mixing in waveguides integrated with graphene oxide. APL Photonics 2018, 3, 120803.

(23) Zhang, Y.; Wu, J.; Yang, Y.; Qu, Y.; Jia, L.; Moein, T.; Jia, B.; Moss, D. J. Enhanced Kerr nonlinearity and nonlinear figure of merit in silicon nanowires integrated with $2 \mathrm{D}$ graphene oxide films. ACS Appl. Mater. Interfaces 2020, 12, 33094-33103.

(24) Liu, H.-L.; Shen, C.-C.; Su, S.-H.; Hsu, C.-L.; Li, M.-Y.; Li, L.-J. Optical properties of monolayer transition metal dichalcogenides probed by spectroscopic ellipsometry. Appl. Phys. Lett. 2014, 105, 201905.

(25) Luke, K.; Okawachi, Y.; Lamont, M. R.; Gaeta, A. L.; Lipson, M. Broadband mid-infrared frequency comb generation in a Si $3 \mathrm{~N} 4$ microresonator. Opt. Lett. 2015, 40, 4823-4826.

(26) Zhang, Y.; Tao, L.; Yi, D.; Xu, J.-b.; Tsang, H. K. Enhanced thermo-optic nonlinearities in a $\mathrm{MoS}_{2}$-on-silicon microring resonator. Appl. Phys. Express 2020, 13, 022004.

(27) Zhang, Y.; Tao, L.; Yi, D.; Xu, J.-B.; Tsang, H. K. Enhanced four-wave mixing with $\mathrm{MoS}_{2}$ on a silicon waveguide. J. Opt. 2020, 22, 025503. 
(28) Deckoff-Jones, S.; Pelgrin, V.; Zhang, J.; Lafforgue, C.; Deniel, L.; Guerber, S.; Ribeiro-Palau, R.; Boeuf, F.; Alonso-Ramos, C.; Vivien, L.; et al. Enhancing SiN waveguide optical nonlinearity via hybrid GaS integration. J. Opt. 2021, 23, 025802.

(29) Torres-Torres, C.; Perea-López, N.; Elías, A. L.; Gutiérrez, H. R.; Cullen, D. A.; Berkdemir, A.; López-Urías, F.; Terrones, H.; Terrones, M. Third order nonlinear optical response exhibited by mono-and few-layers of $\mathrm{WS}_{2}$. 2D Mater. 2016, 3, 021005.

(30) Jung, G.-H.; Yoo, S.; Park, Q.-H. Measuring the optical permittivity of two-dimensional materials without a priori knowledge of electronic transitions. Nanophotonics 2018, 8, 263-270.

(31) Zhang, J.; Pelgrin, V.; Alonso-Ramos, C.; Vivien, L.; He, S.; Cassan, E. Stretching the spectra of Kerr frequency combs with selfadaptive boundary silicon waveguides. Adv. Photonics 2020, 2, 046001.

(32) Tan, D. T.; Agarwal, A. M.; Kimerling, L. C. Nonlinear photonic waveguides for on-chip optical pulse compression. Laser Photonics Rev. 2015, 9, 294-308.

(33) Zhang, S.; Dong, N.; McEvoy, N.; O’Brien, M.; Winters, S.; Berner, N. C.; Yim, C.; Li, Y.; Zhang, X.; Chen, Z.; et al. Direct Observation of Degenerate Two-Photon Absorption and Its Saturation in $\mathrm{WS}_{2}$ and $\mathrm{MoS}_{2}$ Monolayer and Few-Layer Films. ACS Nano 2015, 9, 7142-7150.

(34) Leuthold, J.; Koos, C.; Freude, W. Nonlinear silicon photonics. Nat. Photonics 2010, 4, 535-544.

(35) Wu, J.; Jia, L.; Zhang, Y.; Qu, Y.; Jia, B.; Moss, D. J. Graphene Oxide for Integrated Photonics and Flat Optics. Adv. Mater. 2021, 33, 2006415.

(36) Liu, L.; Xu, K.; Wan, X.; Xu, J.; Wong, C. Y.; Tsang, H. K. Enhanced optical Kerr nonlinearity of $\mathrm{MoS}_{2}$ on silicon waveguides. Photonics Res. 2015, 3, 206-209.

(37) Bai, X.; Li, S.; Das, S.; Du, L.; Dai, Y.; Yao, L.; Raju, R.; Du, M.; Lipsanen, H.; Sun, Z. Single-step chemical vapour deposition of antipyramid $\mathrm{MoS}_{2} / \mathrm{WS}_{2}$ vertical heterostructures. Nanoscale 2021, 13, $4537-4542$.

(38) Weismann, M.; Panoiu, N. C. Theoretical and computational analysis of second-and third-harmonic generation in periodically patterned graphene and transition-metal dichalcogenide monolayers. Phys. Rev. B: Condens. Matter Mater. Phys. 2016, 94, 035435.

(39) Li, Y.; Chernikov, A.; Zhang, X.; Rigosi, A.; Hill, H. M.; Van Der Zande, A. M.; Chenet, D. A.; Shih, E.-M.; Hone, J.; Heinz, T. F. Measurement of the optical dielectric function of monolayer transition-metal dichalcogenides: $\mathrm{MoS}_{2}, \mathrm{MoSe}_{2}, \mathrm{WS}_{2}$, and $\mathrm{WSe}_{2}$. Phys. Rev. B: Condens. Matter Mater. Phys. 2014, 90, 205422.

(40) Malitson, I. H. Interspecimen comparison of the refractive index of fused silica. J. Opt. Soc. Am. 1965, 55, 1205-1209.

(41) Pelgrin, V.; Wang, Y.; Peltier, J.; Alonso-ramos, C.; Vivien, L.; Sun, Z.; Cassan, E. Boosting the SiN nonlinear photonic platform with transition metal dichalcogenide monolayers. arXiv:2105.01467 [physics.optics] 2021, na.

(42) Osgood, R. M., Jr.; Panoiu, N.; Dadap, J.; Liu, X.; Chen, X.; Hsieh, I.-W.; Dulkeith, E.; Green, W.; Vlasov, Y. A. Engineering nonlinearities in nanoscale optical systems: physics and applications in dispersion-engineered silicon nanophotonic wires. Adv. Opt. Photonics 2009, 1, 162-235.

(43) Dudley, J. M.; Genty, G.; Coen, S. Supercontinuum generation in photonic crystal fiber. Rev. Mod. Phys. 2006, 78, 1135.

(44) Hult, J. A fourth-order Runge-Kutta in the interaction picture method for simulating supercontinuum generation in optical fibers. $J$. Lightwave Technol. 2007, 25, 3770-3775. 